\title{
ECONOMÍA DEL CONOCIMIENTO PARA LA SOSTENIBILIDAD
}

\author{
SUSTAINABLE KNOWLEDGE-BASED ECONOMY
}

\section{Pedro César Cantú-Martínez ${ }^{1}$}

\section{Resumen}

El presente manuscrito muestra la trascendencia que posee la economía del conocimiento para el logro de los Objetivos de Desarrollo Sostenible. Se reconoce el conocimiento como un elemento necesario para la búsqueda del bienestar social del ser humano y, además, para la reducción de la pobreza y la falta de equidad social. La economía del conocimiento es el análisis del comportamiento y los hechos relacionados con la aplicación económica del saber. Esto ha llevado a la transformación de las sociedades cuando el conocimiento se convierte en aprendizaje y este se encuentra orientado a resolver los problemas sociales y ambientales.

Palabras claves: economía; conocimiento; desarrollo; sostenibilidad.

\begin{abstract}
This paper shows the importance of knowledge-based economy to achieve sustainable development objectives. Knowledge is recognized as a necessary element for the search for social welfare, the reduction of poverty and the lack of social equity. Knowledge-based economy is behavior analysis and the facts related to the economic application of knowledge. This has led to the transformation of societies when knowledge becomes learning and it is oriented to solve social and environmental problems.
\end{abstract}

Keywords: economy; knowledge; development; sustainability

Doi: http://dx.doi.org/10.15359/eys.22-51.5

Fecha de recepción: 03-11-2016. Fechas de reenvíos: 14-11-2016, 08-11-2016, 27-03-2017, 26-04-2017. Aceptado el 08-05-2017. Publicado el 21-06-2017.

${ }^{1}$ Doctorado en Ciencias Biológicas, Facultad de Ciencias Biológicas, Universidad Autónoma de Nuevo León, San Nicolás de los Garza, N.L., México. Correo electrónico: pedro.cantum@uanl.mx

Pedro César Cantú-Martínez

(c) (i) 8 (2)

Revista Economía y Sociedad by Universidad Nacional is licensed under a CreativeCommons Reconocimiento-NoComercial- 


\section{Introducción}

En el marco global considerado recientemente por los Objetivos de Desarrollo Sostenible (ODS) el impulsor y dispositivo para alcanzar la sostenibilidad y mejorar la calidad de vida de todas las personas lo encontramos en el conocimiento (Cantú-Martínez, 2016a). Esto es ratificado en la Agenda Nacional en Ciencia, Tecnología e Innovación en México (AA. VV., 2012, p. 2), al aseverar que se ha "reconocido repetidamente por muchos líderes políticos, sociales, académicos y empresariales en los países más desarrollados, [que] el fortalecimiento de la inversión en educación, así como en Ciencia, en Tecnología y en la Innovación (CTI) no es un lujo, sino una verdadera necesidad". Lo anterior particularmente porque la utilización del conocimiento se instituye en una circunstancia necesaria y pertinente para el bienestar social de toda colectividad humana (Cantú-Martínez, 2012a; Cantú-Martínez y Moreno, 2012).

Sánchez y Ríos (2011, p. 44) señalan que un desarrollo sostenido que brinde a la sociedad una mejora sustancial de la calidad de vida se apuntala en la "economía del conocimiento". En este ejemplo encontramos el caso de Corea del Sur; estos autores afirman que "las causalidades del crecimiento coreano son una fuerte apuesta e inversión en la educación y la capacitación, el impulso a la innovación científica a través de una política intensa de investigación y desarrollo", entre otras condiciones. Finquelievich (2004) indica que:

(...) ente los elementos comunes a dichas estrategias se destaca la importancia de la investigación y desarrollo (I+D) científicos, la formación de personal altamente calificado, la creación de un medio ambiente rico en iniciativas para sostener la creación y el crecimiento de empresas intensivas en conocimiento e investigación. (p. 7)

Sin lugar a dudas, en las situaciones más exitosas prevalecen políticas de carácter integral cuyo orden es complementario en las cuales se configuran escenarios tan relevantes como la vinculación entre el sector académico y el productivo para hacer uso del mejor capital intelectual humano y del conocimiento de frontera (Cooke y Leydesdorff, 2006; Mallén, 2013).

Rosenberg (2003) menciona que desde hace tiempo nuestro mundo se encuentra en una economía del conocimiento, ya que, después de todo, las instituciones de educación superior son las principales creadoras de este en toda sociedad y además son las principales emisoras; mientras, por otra parte, son las que adicionan nuevos conocimientos a los ya antepuestos. Cómo advierte Cabrero (2014), "se crean relaciones que permiten generar y aprovechar, de manera más eficaz y eficiente, el conocimiento" (p. 36), en el cual se halla la sostenibilidad como componente clave.

En este momento, se torna pertinente y necesario entender qué es sostenibilidad en el marco del desarrollo de toda sociedad y, particularmente, en el concepto de la economía del 2

Pedro César Cantú-Martínez

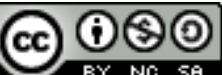

Revista Economía y Sociedad by Universidad Nacional is licensed under a CreativeCommons Reconocimiento-NoComercial- 
conocimiento que tratamos. Referirse a esta noción conlleva una reflexión sobre la forma en que se ha erigido y denominado el desarrollo al que hace alusión el Informe Brundtland, denominado en algunas naciones como "desarrollo sostenible" y en otras como "desarrollo sustentable". En este sentido, Cantú-Martínez (2014) indica que en:

(...) esta situación de orden semántico no media ninguna discrepancia de significado aparente [...], ya que, de manera general, lo que se suscribe simbólicamente en estas expresiones es el precepto de largo alcance y se esboza un amplio rango de acciones sociales, que nos permitan coexistir con una vida plena" (p. 41).

Condición que trasladamos al término de sostenibilidad y sustentabilidad. En el presente manuscrito pretendemos abordar y esclarecer a qué se refiere la economía del conocimiento, explorando sus principios y fundamentos, para finalmente observar su contribución a la sostenibilidad de las sociedades.

\section{¿Qué es el conocimiento?}

Hoy en día el conocimiento ha sido un detonador del desarrollo económico que ha trascendido en una mayor competitividad, particularmente respecto a la productividad, donde esencialmente el conocimiento generado, aunado al componente tecnológico, se han constituido en los elementos claves para incrementar la productividad y dinamizar la economía (Powell y Snellman, 2004; Cabrero, 2014). De acuerdo con Barceló (2001):

El conocimiento surge a partir del Dato, como representación de un grupo de objetos y acontecimientos que se dan en el mundo real [...] una vez que el dato adquiere un determinado significado [...] encontramos un segundo nivel en la información. El flujo de experiencias [...] e información convierten a este segundo nivel en [...] conocimiento. (p. 14)

Por lo tanto, todas las sociedades son generadoras de conocimiento, distinguiéndose cuatro tipos a considerar. Asó, Lundvall y Johnson en 1994 (citados por Barceló, 2001) diferencian entre:

$\checkmark$ Saber-qué: generalmente hace referencia a hechos y a proposiciones ciertas.

$\checkmark$ Saber-por qué: hace referencia al conjunto de estructuras explicativas de los principios y leyes de la naturaleza; es decir, al entendimiento.

$\checkmark$ Saber-cómo: se refiere a las capacidades y habilidades para hacer algo.

$\checkmark$ Saber-quién: engloba la información sobre quién sabe qué y quién sabe cómo hacer qué.

Las dos primeras menciones corresponden al conocimiento explícito o codificado; y las dos restantes, al conocimiento de carácter tácito o no codificado. El primero es aquel que se

Pedro César Cantú-Martínez

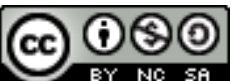

Revista Economía y Sociedad by Universidad Nacional is licensed under a CreativeCommons Reconocimiento-NoComercial- 
adquiere mediante la utilización de bases de datos, o bien, mediante la lectura de libros, entre algunos ejemplos; mientras el segundo conocimiento está sustentado primordialmente en la experiencia y lo ostentan las personas (Barceló, 2001). De tal manera que el conocimiento es el agrupamiento de representaciones abstractas producto de la adquisición o de la experiencia alcanzada en función de la observación de la realidad. Esta información, posteriormente, la traducimos en acción para modificar o recrear la realidad (Luque, 1993; Potts, 2001; CantúMartínez y Waliszewski, 2012; Kanuma, Butera y Butera, 2015). Así, se transita del saber al hacer mediante el conocimiento.

\section{Principios de la economía del conocimiento}

La relevancia del conocimiento como un insumo único para la sociedad se ha constituido gradualmente en un tópico de sumo interés, esencialmente cuando las naciones industrializadas observaron en ello un factor determinante para el desarrollo económico, como ha sucedido "desde la segunda revolución industrial [donde] la vinculación entre el conocimiento científico y las tecnologías productivas es evidente" (Vilaseca, Torrent y Díaz, 2002, p. 5). En este sentido, la Organización para la Cooperación Económica y Desarrollo esgrimió, desde el año 1996, la conveniencia de alinear las políticas de ciencia, tecnología e industria hacia la búsqueda del máximo bienestar social y el rendimiento productivo; a esto lo denominó "economías basadas en conocimiento" (Organisation for Economic Cooperation and Development, 1996). De acuerdo con Sánchez y Ríos (2011):

Una economía del conocimiento es aquella en la que el conocimiento es un activo más importante que los bienes de capital y mano de obra, y donde la cantidad y sofisticación del conocimiento que permea en las actividades económicas y sociales, llega a niveles muy altos. (p. 46)

Esto es, además de reconocer el valor de invertir para fortalecer y acrecentar el conocimiento, también se estima como sumamente apreciable la distribución del conocimiento mediante redes sociales tanto formales como informales, para mejorar el repartimiento y ejercicio económico en la sociedad y así favorecer un bienestar social masivo (Canales y León, 2013; Guzmán, 2016), lo que conlleva a que, en el escenario de las políticas públicas, estas se centren especialmente en la actualización del capital humano y en la promoción de la obtención de mejores habilidades para aprender.

Sánchez y Ríos (2011) indican que para el Banco Mundial el conocimiento cuenta con cuatro puntales que dan soporte a la economía del conocimiento, estos son: a) educación y la capacitación de la fuerza laboral, b) adecuados servicios para la propagación y utilización de la información y el conocimiento, c) contar con sistemas de innovación en los que se plasme la vinculación entre las instituciones de educación superior y las empresas, d) un régimen institucional de estímulos financieros que permita la concesión de recursos de forma eficaz para 4

Pedro César Cantú-Martínez

(c) (i) 8 (2)

Revista Economía y Sociedad by Universidad Nacional is licensed under a CreativeCommons Reconocimiento-NoComercial- 
afirmar la práctica eficiente del conocimiento. En todo lo anterior resulta notorio, como aluden Moreno, Pelayo y Vaca (2007):

(...) que conocimiento y aprendizaje deben ir unidos: el conocimiento es el recurso y el aprendizaje es la capacidad para obtenerlo, crearlo y usarlo. Ambos son inseparables. Por tanto, el aprendizaje puede entenderse como una acción que, en sentido amplio, toma el conocimiento como input y genera nuevo conocimiento. (p. 3611)

Ahora bien, de acuerdo con Cabrero (2014), consolidar estos puntales:

Brinda a los países un ambiente idóneo para que los tres actores principales interactúen dentro de una economía basada en el conocimiento: las universidades y centros de investigación -encargados de formar mano de obra calificada y crear conocimiento-; las empresas -que absorben el conocimiento y a los egresados de las universidades, a la vez que realizan innovaciones-; y el sector público o gobierno -orientado a encaminar las políticas públicas en materia de ciencia, tecnología e innovación (CTI) para facilitar la relación entre los sectores educativo y productivo, y a llenar los vacíos que impidan una interacción efectiva entre ambos-. (p. 36)

A este modelo antes citado se le denomina como la "Triple Hélice", que es donde se inspira el proceso de innovación y que permite afianzar la capitalización del conocimiento, vinculando distintas disciplinas y conocimientos, además de fomentar substancialmente las relaciones de colaboración entre los tres actores, instituciones de educación superior, empresa y gobierno (Chang, 2010; Leydesdorff, 2010). Esto ha conllevado en gran parte a una transformación de las sociedades, concatenada hoy en día "a la revolución biotecnológica, el avance científico y la era de la información" (González, 2006, p. 11), que despunta los alcances e influencia de los sucesos tecnocientíficos en la humanidad y de las sucesión de cambios que hurgan en la cotidianidad de la vida diaria (Gentzoglanis, 2000; Cantú-Martínez, 2012b; Rodríguez-Ponce y Pedraja-Rejas, 2015). En este sentido, Canales y León (2013, p. 62) mencionan que es muy relevante apreciar "que si bien el conocimiento siempre ha estado presente en las transformaciones productivas, en la actualidad está siendo creado y transferido con mayor rapidez, en contraste con lo que ocurría en décadas pasadas".

Lo antes mencionado se puede observar claramente en dos dimensiones del conocimiento, de acuerdo con Bernal (2008): “i) la dimensión global, que abarca el proceso de producción, transferencia y uso del conocimiento fuera de su contexto (codificado) y ii) la dimensión local, que incluye el proceso de aprendizaje y sedimentación, cuando el conocimiento se arraiga" ( $p$. 25). 


\section{Economía del conocimiento en la sostenibilidad}

La transformación de la realidad que se suscita en derredor nuestro, en este período de modernidad, es sumamente complicada ya que ha conllevado progreso por una parte, como también dificultades de orden social. En la actualidad, Cantú-Martínez (2012a) sostiene que:

(...) la realidad es bastante compleja, y su resultado es el reflejo en conjunto de la concatenación de la formación de recursos humanos, de la disponibilidad del saber científico y técnico, de las condiciones y aplicabilidad práctica y económica de la misma, así como de la demanda social que en materia de conocimiento tecnocientífico lograra existir. (p. 30)

Esto evidencia que los problemas en las esferas económica, social y ambiental son inconvenientes primordialmente humanos, que denotan lo importante del conocimiento existente para evaluar, resolver y emplear este mediante aplicaciones concretas al servicio de la sociedad, con la finalidad de reflexionar en las estrategias generales de desarrollo económico para promover claramente los principios de la sostenibilidad (Fundación Friedrich Naumann y Fundación Este País, 2005; Cantú-Martínez, 2015a).

De acuerdo con lo antes mencionado, la economía del conocimiento se yergue como una vía para fomentar un desarrollo sostenible con un carácter continuo, como una ruta para sufragar la escasez y carencia de recursos y, con esto, cerrar las brechas existentes, favoreciendo la equidad social y eliminación de la pobreza (Chen y Dahlman, 2005). En este sentido, Holling (2000) advierte, en el marco de lo que considera un sistema de correspondencia entre la simplicidad y la complejidad, que la sostenibilidad nos conduce a la capacidad de encontrar una armonía y adaptación permanente entre las dimensiones social, económica y ambiental, mientras que el desarrollo denota el proceso de crear y mantener vigentes las oportunidades entre las dimensiones antes mencionadas. Es así que se construye el desarrollo sostenible, como la vía social para concebir, por una parte, capacidades de adaptación y, por otra, labrar oportunidades para la continuidad de la sociedad humana. Es más, esta postura requiere, conforme Torrent-Sellens (2016, p. 30):

(...) articular nuevas funciones de comportamiento y nuevas métricas de la economía. De hecho, y como ya ha sucedido en otras fases de la historia de la economía, la economía del conocimiento necesita articular un nuevo paradigma científico, que explique con más fidelidad la realidad económica.

Un esfuerzo fehaciente de lo antes señalado es la presentación de la Agenda Ciudadana en Iberoamérica Ciencia, Tecnología e Innovación, preparada por el Comité Organizador Iberoamericano Colombia-España-México, en la reunión celebrada el 6 y 7 de octubre del 2016 en Cartagena de Indias, Colombia, a la que asistieron los ministros y autoridades de Ciencia, 6

Pedro César Cantú-Martínez

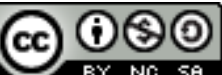

Revista Economía y Sociedad by Universidad Nacional is licensed under a CreativeCommons Reconocimiento-NoComercial- 
Tecnología e Innovación. Esta agenda responde al planteamiento de los Objetivos de Desarrollo Sostenible de la Organización de las Naciones Unidas (Cantú-Martínez, 2016b), y en ella se ocupa de los primeros siete temas planteados en estos: Energía; Seguridad Alimentaria; Sociedad Digital; Educación; Medio Ambiente; Cambio Climático y Sustentabilidad; y Agua y Salud. El octavo tema fue seleccionado por cada país, por ejemplo, Colombia eligió Paz, mientras que España y México, Envejecimiento (Inzunza, 2016).

El propósito es que esta agenda se constituya en un documento orientador que induzca su aplicación a reflexiones, generación de ideas, creación de patrimonio, progreso social, acopio y regeneración del conocimiento y que brinde, además, oportunidades para mejorar las condiciones de vida en toda sociedad. Según señaló José Franco, miembro de la Comisión Coordinadora en Iberoamérica durante la reunión: "en este ejercicio estamos apostándole al gran valor de nuestra región y sus culturas. A nuestro futuro, porque tenemos los recursos naturales, materiales y sobre todo el invaluable talento, creatividad y determinación de nuestra gente" (Inzunza, 2016, p. 27). De cumplirse la Agenda Ciudadana, se favorecerá en definitiva al desarrollo sostenible basado ahora en el conocimiento, desde su lanzamiento por el Informe Brundtland (Cantú-Martínez, 2008; Cantú-Martínez, 2015b).

Es importante reconocer que las economías basadas en el conocimiento, aunque contribuyen al desarrollo sostenible, requieren de grandes cantidades de recursos que deben ser empleados en la educación, investigación, innovación científica y, posteriormente, para la transferencia social y aplicación; además, es esencial contar con un marco de referencia con cierto nivel de desarrollo industrial existente. Ramos (2015) advierte lo siguiente:

No es casual que las economías más fuertes en conocimiento, como Estados Unidos, Japón, Alemania y recientemente Corea o China, sean las que tienen niveles más altos de consumo de energía y materiales, así como los sectores industriales más desarrollados. (p. 9)

\section{Conclusiones}

Desde la promulgación del Informe Brundtland en 1992, las autoridades gubernamentales de todas las naciones han comprendido que no se debe considerar únicamente las cuestiones económicas, sino también las de carácter social y ambiental para mejorar las condiciones de vida y satisfacer las demandas de orden social. En el contexto de la actualidad, los principios que rigen el concierto internacional para allegarse el desarrollo sostenible se fundan en el conocimiento. Con estos nuevos marcos referenciales de la economía del conocimiento se ha introducido este como la clave para resolver los problemas que nos aquejan, ya que es observado como insumo y como un bien de creciente cuantía y significación, al estar orientado hacia la búsqueda del respeto de los derechos humanos, la satisfacción de las necesidades sociales y la conservación del entorno.

Pedro César Cantú-Martínez

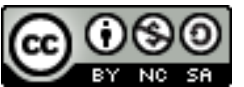

Revista Economía y Sociedad by Universidad Nacional is licensed under a CreativeCommons Reconocimiento-NoComercial- 
Cabe recordar que, según se encuentra documentado, hasta hace unos años el desarrollo económico de un país estribaba en la capacidad para generar riqueza en el tiempo, que se percibía como el hecho de acumular capital y trabajo únicamente mediante el acto de incrementar la productividad. Además, de forma particular se pensaba que una nación con amplios recursos económicos y naturales poseía ventajas sobre otra con recursos escasos (Marquina y Rozga, 2015). No obstante, hoy en día estos pensamientos se han ido abandonando paulatinamente al examinarse en las distintas naciones que la ventaja reside sustancialmente en la manera eficiente de utilizar los recursos con los que se cuenta, empleando el conocimiento como la totalidad de factores que toman parte en la manufactura de otros bienes. A partir de esto, se puede hacer hincapié en que el conocimiento se traduce en labores humanas emanadas de las interacciones surgidas entre las sociedades con un carácter dinámico que crean riqueza, bienestar social y también la oportunidad acceder a la sostenibilidad que tanto se anhela.

\section{Referencias}

AA. VV. (2012). Hacia una Agenda Nacional en Ciencia, Tecnología e Innovación. México. Recuperado de www.foroconsultivo.org.mx/documentos/agenda nal cti extenso 260912.pdf

Barceló, M. (2001) (Dir.) Hacia una economía del conocimiento. Madrid: ESIC-EditorialPricewaterhouseCoopers. Recuperado de https://books.google.com.mx/books?id=jn3FdhLNuBIC\&printsec=frontcover\&source =gbs ge summary $r \& c a d=0 \# v=$ onepage $\& q \& f=$ false

Bernal, J.L. (2008). El desempeño de la economía del conocimiento a nivel regional; un análisis empírico del caso de México. (Tesis de Maestría). Universidad Autónoma Metropolitana. Cd. de México, México. Recuperado de http://tesiuami.izt.uam.mx/uam/aspuam/test.php?asesor=JUAN\%20CASTAINGTS\%2 $\underline{\text { OTEILLERY }}$

Cabrero, E. (2014). Ciudades del conocimiento, ciudades sustentables. Ciencia, (OctubreDiciembre), 34-39. Recuperado de http://www.revistaciencia.amc.edu.mx/images/revista/65 4/PDF/CiudadConocimie nto.pdf

Canales, R. y León, D. (2013). Redes y conocimiento: una propuesta integradora en el marco de la Economía del Conocimiento. Explanans, 2(1), 59-74. Recuperado de https://www.researchgate.net/publication/275521253 Redes_y conocimiento una _propuesta integradora en el marco de la Economia del Conocimiento

8

Pedro César Cantú-Martínez

(c) (i) 8 (2)

Revista Economía y Sociedad by Universidad Nacional is licensed under a CreativeCommons Reconocimiento-NoComercial- 
Cantú-Martínez, P.C. (2008). Desarrollo Sustentable: Conceptos y Reflexiones. México: Ed. Universidad Autónoma de Nuevo León. Recuperado de https://sites.google.com/site/drpedroccantumartinez/home/libros-publicados

Cantú-Martínez, P.C. (2012a). Discurrir y apropiamiento de la ciencia por la sociedad. En Cantú Martínez, P.C. (Ed.) Sustentabilidad Científica. Introversión sobre la Ciencia, Conciencia y Racionalidad Social, 25-32. México: Clave Editorial y Universidad Autónoma de Nuevo León. Recuperado de https://sites.google.com/site/drpedroccantumartinez/home/libros-publicados

Cantú-Martínez, P.C. (2012b). Ética en la sociedad tecnocientífica. En Cantú Martínez, P.C. (Ed.) Sustentabilidad Científica. Introversión sobre la Ciencia, Conciencia y Racionalidad Social, 33-44. México: Clave Editorial y Universidad Autónoma de Nuevo León. Recuperado de https://sites.google.com/site/drpedroccantumartinez/home/librospublicados

Cantú-Martínez, P.C. (2014). Educación ambiental y la escuela como espacio educativo para la promoción de la sustentabilidad. Revista Electrónica Educare, 18(3), 39-52. Doi: http://dx.doi.org/10.15359/ree.18-3.3

Cantú-Martínez, P.C. (2015a). Desarrollo sustentable. Antes y después de Río +20. México: Universidad Autónoma de Nuevo León - Organización Panamericana de la Salud. Recuperado de https://sites.google.com/site/drpedroccantumartinez/home/librospublicados

Cantú-Martínez, P.C. (2015b). Ascenso del desarrollo sustentable. De Estocolmo a Río +20 Ciencia UANL, 18(75), 33-39. Recuperado de http://cienciauanl.uanl.mx/?p=4827

Cantú-Martínez, P.C. (2016a). Implicaciones de los Objetivos del Desarrollo Sustentable. Ciencia UANL, 19(80), 30-34. Recuperado de http://cienciauanl.uanl.mx/?p=5997

Cantú-Martínez, P.C. (2016b). Los nuevos desafíos del desarrollo sustentable hacia 2030. Ciencia UANL, 19(78), 27-32. Recuperado de http://eprints.uanl.mx/11001/

Cantú-Martínez, P.C. y Moreno, D. (2012). El conflicto social de la ciencia En Cantú-Martínez, P.C. (Ed.) Sustentabilidad Científica. Introversión sobre la Ciencia, Conciencia y Racionalidad Social, 53-59. México: Clave Editorial y Universidad Autónoma de Nuevo León. Recuperado de https://sites.google.com/site/drpedroccantumartinez/home/libros-publicados 
Cantú-Martínez, P.C. y Waliszewski, S.M. (2012). Ser y saber tecnocientíficos. En Cantú Martínez, P.C. (Ed.) Sustentabilidad Científica. Introversión sobre la Ciencia, Conciencia y Racionalidad Social, 45-52. México: Clave Editorial y Universidad Autónoma de Nuevo León. Recuperado de https://sites.google.com/site/drpedroccantumartinez/home/libros-publicados

Chang, H.G. (2010). El modelo de la triple hélice como un medio para la vinculación entre la universidad y empresa. Revista Nacional de Administración, 1(1), 85-94. Doi: http://dx.doi.org/10.22458/rna.v1i1.286

Chen, D.H.C. y Dahlman, K.J. (2005). The Knowledge Economy, the KAM Methodology and World Bank Operations (Working Paper 35867). Recuperado de http://documents.worldbank.org/curated/en/695211468153873436/Theknowledge-economy-the-KAM-methodology-and-World-Bank-operations

Cooke, P. y Leydesdorff, L. (2006). Regional Development in the Knowledge-Based Economy: The Construction of Advantage. Journal of Technology Transfer, 31(1), 5-15. Recuperado de http://link.springer.com/article/10.1007/s10961-005-5009-3

Finquelievich, S. (2004). La sociedad civil en la economía del conocimiento: TICs y desarrollo socio-económico. Buenos Aires: Instituto de Investigaciones Gino Germani, Facultad de Ciencias Sociales-UBA. Recuperado de http://bibliotecavirtual.clacso.org.ar/Argentina/iigg-uba/20100719121159/dt40.pdf

Fundación Naumann y Fundación Este País (2005). México ante el reto de la economía del conocimiento. México: FNST/FES. Recuperado de http://archivo.estepais.com/inicio/historicos/174/20 suplemento mexico\%20ante \%20el\%20reto.pdf

Gentzoglanis, A. (2000). Innovation and Growth in the Knowledge-based Economy. Ciencia Ergo Sum, 221-228. 2(3), Recuperado de http://cienciaergosum.uaemex.mx/index.php/ergosum/article/view/3864

González, G. (2006). La Economía del Conocimiento y las relaciones de oferta y demanda laboral (Tesis de Licenciatura). Recuperado de http://tesiuami.izt.uam.mx/uam/aspuam/presentatesis.php?recno=13476\&docs=U $\underline{\text { AMI13476.PDF }}$

10 
Guzmán, T. (2016). Proceso de diseño de un modelo de educación a distancia como estrategia de innovación educativa para la economía del conocimiento. Edutec-e Revista Electrónica de Tecnología Educativa, (55), 1-15. Doi: http://dx.doi.org/10.21556/edutec.2016.55.729

Holling, C.S. (2000). Theories for sustainable futures. Conservation Ecology, 4(2), 7. Recuperado de http://www.consecol.org/vol4/iss2/art7/

Inzunza, A. (6 de octubre 2016). Presentan Agenda Ciudadana para Iberoamérica. Forum Noticias del Foro Consultivo, 18, 19-34. Recuperado de http://www.foroconsultivo.org.mx/FCCYT3/index.php/sala-de-prensavirtual/boletines-de-prensa/5297-presentan-agenda-ciudadana-para-iberoamerica

Kanuma, N., Butera, V. y Butera, A. (2015). Enhancing a sustainable knowledge based economy through private sector led-development: A Case Study of Private Higher Learning Institutions in Rwanda. East African Journal of Science and Technology, 5 (1), 78-94. Recuperado de http://eajournal.unilak.ac.rw/Vol\%205\%20lssue\%201/Paper6.pdf

Laydesdorff, L. (2010). The knowledge-based economy and the triple helix model. Annual Review of Information Science and Technology, 44, 1-54. Recuperado de https://arxiv.org/ftp/arxiv/papers/1201/1201.4553.pdf

Luque, J. (1993). El conocimiento. Sevilla: Universidad de Sevilla. Recuperado de http://personal.us.es/jluque/Libros\%20y\%20apuntes/1994\%20Conocimiento.pdf

Mallén, C. (2013). Conocimiento para el crecimiento sustentable de México. Revista Mexicana de Ciencias Forestales, 4(15), 4-6. Recuperado de http://cienciasforestales.inifap.gob.mx/editorial/index.php/Forestales/article/view/2734/22 $\underline{98}$

Marquina, M.L. y Rozga, R.E. (2015). La economía del conocimiento: perspectivas urbanoregionales. Proyección, IX, 6-30. Recuperado de http://www.proyeccionrevista.com.ar/revistas/proyeccion-n-18/la-economia-delconocimiento-perspectivas-urbano-regionales 
Moreno, M., Pelayo, Y. y Vaca, R. (2007). La gestión interna del conocimiento en las organizaciones: un modelo de indicadores para el control de su gestión. En Ayala Calvo, J.C. (Coord.) Conocimiento, innovación y emprendedores: camino al futuro. (3610-3629). España: Universidad de la Rioja. Recuperado de https://publicaciones.unirioja.es/catalogo/online/Jornadas_Gestion_cientifica.shtml http://dialnet.unirioja.es/servlet/fichero articulo?codigo=2234967

Organization for Economic Co-operation and Development. (1996). The knowledge based economy. París: OECD. Recuperado de https://www.oecd.org/sti/scitech/1913021.pdf

Potts, J. (2001). Knowledge and Markets. Journal of Evolutionary Economics, 11 (4), 413-431. Recuperado de https://link.springer.com/article/10.1007/PL00003865

Powell, W.W. y Snellman, K. (2004). The knowledge economy. Annual Review of Sociology, 30, 199-220. Recuperado de https://scholar.harvard.edu/

Ramos, J. (2015). Base material de la economía del conocimiento. América Latina en Movimiento, 507, 8-10. Recuperado de http://www.alainet.org/sites/default/files/alai507w.pdf

Rodríguez-Ponce, E. y Pedraja-Rejas, L. (2015). The role of regional universities in the Chilean decentralization process: A view from the knowledge economy. Ingeniare Revista Chilena de Ingeniería, 23 (4), 490-492. Recuperado de http://www.scielo.cl/scielo.php?script=sci arttext\&pid=S071833052015000400001\&lng=en\&nrm=iso\&tlng=en

Rosenberg, N. (2003). Conocimiento e innovación como herramientas para alcanzar el desarrollo económico: ¿acaso las universidades deben ser instituciones económicas? En Briceño, M.A. (Comp.) Universidad, sector productivo y sustentabilidad, 83-96. Caracas. Universidad Central de Venezuela. Recuperado de https://books.google.co.cr/books?id=CnllzDmlAQcC\&printsec=frontcover\&dq=isbn: 9800019669\&hl=es-419\&sa=X\&redir esc=y\#v=onepage\&q\&f=false

Sánchez, C. y Ríos, H. (2011). La economía del conocimiento como base del crecimiento económico en México. Enl@ce Revista Venezolana de Información, Tecnología y Conocimiento, 8 (2), 43-60. Recuperado de http://www.redalyc.org/pdf/823/82319126004.pdf

12 
Torrent-Sellens, J. (2016). La economía del conocimiento y el conocimiento de la economía. Oikonomics Revista de Economía, Empresa y Sociedad, 5, 26-32. Recuperado de http://www.uoc.edu/portal/es/estudis arees/economia_empresa/novetats/noticia 017.html

Vilaseca, J., Torrent, J. y Díaz, A. (2002). La economía del conocimiento: paradigma tecnológico y cambio estructural. España: UOC. Recuperado de http://www.uoc.edu/in3/dt/20007/20007.pdf 